\title{
Úlcera escleral secundaria a quemadura química por pila de reloj y cuerpo extraño retenido en fondo de saco conjuntival inferior. Caso clínico
}

\author{
KATHERINE KOCH V. ${ }^{1}$, THAMARA AZCARATE M. ${ }^{2}$, DEYKALEJANDRA ROMERO O. ${ }^{3}$, \\ MARÍA OTILIA SUÁREZ Z. ${ }^{4}$, FANNY LEONARDI $\mathrm{C}^{5}$ \\ 1. Residente Universidad de Carabobo. Sede Aragua. Servicio de Pediatría. Hospital Central de Maracay. \\ Estado Aragua, Venezuela. \\ 2. Pediatra Puericultor. Servicio de Pediatría III del Hospital Central de Maracay, Universidad de Carabobo. Sede Aragua. \\ Maracay, Estado Aragua. Venezuela. \\ 3. Universidad de Carabobo. Sede Aragua. Servicio de Pediatría. Hospital Central de Maracay. Estado Aragua, Venezuela. \\ 4. Pediatra Puericultor. Especialista Adscrita al IVSS Centro Ambulatorio "Dr. Germán Quintero". Estado Miranda, \\ Los Teques. \\ 5. Médico Rural. Hospital Municipal del Junquito Distrito Capital. Venezuela.
}

\section{ABSTRACT \\ Scleral ulcer secondary to chemical burn caused by watch battery and retained foreign body in conjunctival sac inside. Case report}

Introduction: Approximately one-third of childhood blindness is due to trauma, representing the leading cause of visual impairment and monocular blindness in children. Case report: The patient is a 2 year old preschool boy, who presented scleral ulcer in his left eye secondary to chemical burn caused by watch battery. The reason for seeking medical care was difficult healing caused by a mineral foreign body retained in the lower conjunctival sac after the patient came in contact with detergent one month before the pediatric consultation. Discussion: An unusual case of scleral ulcer was reported. So far, no other similar cases have been reported. The diagnosis process and the delay for the removal of the foreign body are emphasized in this serious ophthalmologic emergency.

(Key words: Chemical burn, scleral ulcer, ocular trauma).

Rev Chil Pediatr 2012; 83 (6): 582-586

\section{RESUMEN}

Introducción: Aproximadamente un tercio de las cegueras infantiles son debidas a los traumatismos, siendo la principal causa de déficit visual y ceguera monocular en niños. Caso clínico: Se reporta el caso de un preescolar masculino de 2 años de edad quien presentó úlcera escleral en ojo izquierdo secundaria a quemadura química por pila de reloj cuyo motivo de consulta fue contacto con detergente, con cicatrización dificultosa debido a cuerpo extraño mineral retenido en fondo de saco conjuntival inferior durante aproximadamente un

Recibido el 3 de diciembre de 2011, devuelto para corregir el 9 de enero de 2012, segunda versión el 25 de marzo de 2012 , aceptado para publicación el 9 de julio de 2012.

Este trabajo cumple con los requisitos sobre consentimiento /asentimiento informado, comité de ética, financiamiento, estudios animales y sobre la ausencia de conflictos de intereses según corresponda.

Correspondencia a:

Dra. Katherine Koch Villegas

E-mail: drakatherinekoch@gmail.com 
mes, contando desde el ingreso al área pediátrica. Conclusión: Se reporta un caso inusual de úlcera escleral. Hasta el momento no se han reportado otros casos de úlcera escleral secundaria a quemadura química por pila de reloj y por objetos retenidos en fondo de saco conjuntival inferior, resaltando el manejo diagnóstico y la demora en la extracción del cuerpo extraño, representando una emergencia oftalmológica.

(Palabras clave: Quemadura química, úlcera escleral, trauma ocular).

Rev Chil Pediatr 2012; 83 (6): 582-586

\section{Introducción}

Aproximadamente un tercio de las cegueras infantiles son debidas a los traumatismos, siendo la principal causa de déficit visual y ceguera monocular en niños ${ }^{1}$. Un $25 \%$ de ellos corresponde a niños ${ }^{2}$, es más frecuente en el sexo masculino con una relación de 4 a $1^{3}$. Predomina en el grupo etáreo de 5 a 14 años en un $85 \%$ y es raro en menores con un $4 \%{ }^{4}$.

Los traumatismos oculares son causados en un 59\% por deportes o juegos, un $37 \%$ con golpes y caídas accidentales y un $12 \%$ corresponde a lesiones provocadas por la mano o el pie de otro niño ${ }^{1}$, siendo la mayoría de estos traumatismos de globo cerrado $(42 \%)^{4,5}$, afectando la conjuntiva, córnea y esclera $(74 \%)^{5}$. Los cuerpos extraños corresponden a un $4 \%$ de los traumas abiertos 5 , y la principal lesión de polo anterior por sustancias químicas es la corneal, siendo la esclerótica menos afectada ${ }^{4}$.

En un estudio realizado por Zaghen ${ }^{6}$ et al. en Barquisimeto, Venezuela; sobre traumatismos oculares con criterios de hospitalización, se encontró que la edad predominante fue entre 7 y 9 años (35\%), seguido del grupo de 4 a 6 años (31\%), observándose mayor frecuencia en el sexo masculino (72\%). La principal causa fueron los accidentes domésticos $(62 \%)$ y los juegos (15\%). La lesión ocular más frecuente fue el hifema $(36 \%)$, la herida corneal con un $32 \%$ y menos frecuente las heridas esclerales $(4 \%)$. El objetivo es reportar el caso de un preescolar que consulta por úlcera escleral en ojo izquierdo secundaria a quemadura química por pila de reloj y a cuerpo extraño retenido en fondo de saco conjuntival inferior.

\section{Caso clínico}

Preescolar masculino de 2 años de edad natural y procedente de Cagua, Estado Aragua
Venezuela, quien fue llevado a centro de Atención Primaria en mayo de 2010, cuando posterior a contacto ocular con detergente, presentó edema bipalpebral y limitación para la apertura ocular, realizándose lavado ocular y egresando con antibióticos tópicos; posteriormente, se asoció secreción amarillenta en ojo izquierdo, por lo que fue llevado en múltiples oportunidades a consulta médica, manteniendo tratamiento. Por persistencia de síntomas, fue llevado a oftalmólogo en centro privado, quien evalúa y refiere a Hospital Central de Maracay.

Al examen oftalmológico de ingreso se evidenció en ojo izquierdo, párpado con edema severo que no permitió apertura ocular, pestañas y fondo de saco conjuntival con abundante secreción verdosa, adelgazamiento de conjuntiva y esclerótica, con protrusión de tejido uveal; no se logró evaluar el fondo de ojo. Se planteó adelgazamiento escleral posterior a quemadura química complicado con protrusión de tejido uveal. La hematología y las prueba de coagulación estaban dentro de rangos normales. Se inició tratamiento con moxifloxacina tópica, lagrimas artificiales, y antibioticoterapia endovenosa: oxacilina (150 $\mathrm{mg} / \mathrm{kg} /$ día), cloranfenicol (100 mg/kg/día) y amikacina (15 mg/kg/día). A las 24 h se realizó nueva evaluación oftalmológica con hallazgos similares al ingreso, asociándose adelgazamiento de conjuntiva y esclera en tercio medio de conjuntiva bulbar de aproximadamente $1 \mathrm{x}$ $1,5 \mathrm{~cm}$ de diámetro, con protrusión de úvea en tercio inferior de epitelio escleral de $3 \times 4 \mathrm{~mm}$, y cornea transparente; no se realizó fondo de ojo por la poca colaboración del paciente.

Dos días posterior al ingreso, se realizó revisión ocular bajo anestesia de ojo izquierdo visualizando secreción verdosa abundante, hiperemia conjuntival, quemosis, cornea transparente, y cámara anterior indemne; se realizó 


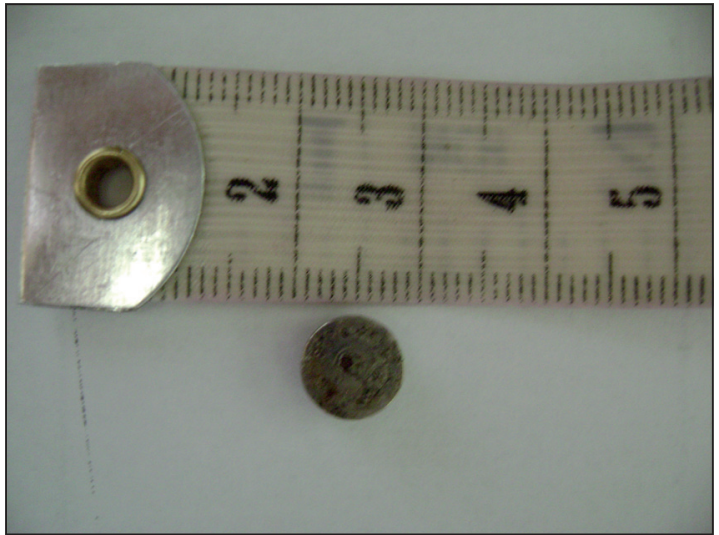

Figura 1. Cuerpo extraño extraído de conjuntiva y esclera ojo izquierdo.

apertura ocular con separadores, evidenciando la presencia de cuerpo extraño en conjuntiva y esclera de ojo izquierdo, tipo pila de reloj de $1 \times 1 \mathrm{~cm}$ la cual se extrae; se diagnosticó úlcera escleral secundaria a cuerpo extraño (figura 1).

El preescolar fue evaluado en 5 oportunidades por servicio de oftalmología, quienes en vista de presencia de úlcera escleral que no cicatrizaba, plantearon colocación de parche en esclera, realizándose estudios preoperatorios, que arrojaron el 23/05/10 prolongación de Tiempo de Tromboplastina Parcial (diferencial $+15,4$ segundos) motivo por el cual recibió plasma fresco congelado previo acto quirúrgico, y en vista de persistir prolongado, se difirió la cirugía.

Se reevaluó en junio de 2010 evidenciando discreta perforación escleral, con consistencia dura a la palpación de parpado inferior izquierdo, por lo que se indicó TAC, donde se visualizó cuerpo extraño en fondo de saco conjuntival (figura 2). Se retiró el segundo cuerpo extraño con hallazgo quirúrgico de fragmento mineral el cual pulveriza en arena durante su extracción. Se observó posterior cicatrización de la esclerótica (figura 3), egresando del área pediátrica en junio de 2010 en su trigésimo tercer día de hospitalización.

Preescolar se reevalúa ambulatoriamente en agosto de 2010, con cicatrización de la úlcera escleral y casi completa reepitelización de esclera.

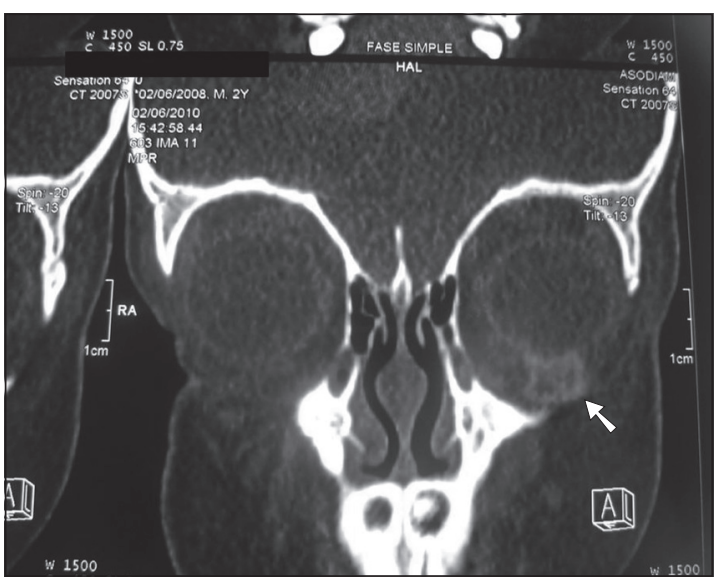

Figura 2. Tomografía axial computada mostrando cuerpo extraño en fondo de saco conjuntival (flecha).

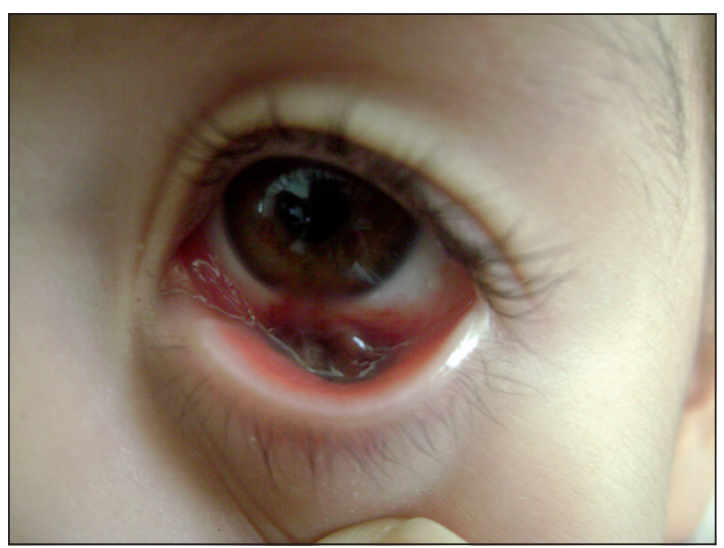

Figura 3. Cicatriz residual en esclera ojo izquierdo.

\section{Discusión}

En el caso reportado de preescolar masculino de 2 años de edad, se describe la presencia de ulceración escleral como consecuencia de quemadura química por pila de reloj y cuerpo extraño tipo fragmento mineral, manteniéndose indemne la cornea. Es importante destacar que la úlcera pudo ser por quemadura química, fricción o ambos mecanismos, considerando que la pila de reloj contiene álcalis y la presencia del cuerpo extraño condiciona el trauma ocular abierto; no se descartan las tres posibilidades siendo la más factible la tercera. Así mismo, la persistencia de la úlcera estaría causada por la presencia del segundo cuerpo extraño de tipo fragmento mineral, y se confir- 
ma por el hecho de la mejoría clínica posterior extracción.

Es probable que no se haya visualizado fragmento mineral debido al edema del tejido palpebral y conjuntival durante los primeros exámenes oftalmológicos; no se descarta la posibilidad de que el preescolar se haya introducido posteriormente el cuerpo extraño, detalle no descrito pues durante el interrogatorio abuela materna al cuidado y preescolar negaron la acción. Se descarta lesión corneal al no evidenciar opacidad de esta, hallazgo inicial en quemaduras clínica, tampoco se visualizó signos de erosión corneal ${ }^{4}$.

La mayoría de los cuerpos extraños superficiales corneales son metálicos, seguido en frecuencia por fragmentos de piedra y madera, siendo vectores importantes de contaminación ${ }^{7}$, hallazgos observados en el caso descrito, así como la necesidad del uso de antibióticos tópicos y sistémicos, por secreción verdosa como hallazgo sugestivo de infección al examen oftalmológico de ingreso.

La exploración física está llena de dificultades y debe ser realizada por personal experto. La ansiedad y el dolor del paciente dificultan la cooperación debiendo efectuarse bajo sedación o anestesia general ${ }^{8}$; la prueba imagenológica inicial en caso de cuerpo extraño elección es la radiografía simple, la ecografía no se recomienda en caso de perforación y probable expulsión de cuerpo extraño, sugiriendo el uso de la TAC en esas circunstancias ${ }^{8}$. En este caso se realizó exploración oftalmológica bajo anestesia, permitiendo la extracción del primer cuerpo extraño; Se realizó TAC de órbita (figura 3) por no mejoría de la úlcera, visualizándose imagen radiopaca en fondo de saco conjuntival inferior, con la posterior extracción de cuerpo extraño tipo fragmento mineral.

Es de suma importancia el lavado ocular como tratamiento inicial en las quemaduras por álcalis y sustancias químicas, procedimiento realizado en Centro de Atención Primaria que recibió al paciente, la meta terapéutica es neutralizar el pH del ojo, sin embargo, en estos centros no se cuenta con papel tornasol para determinar el $\mathrm{pH}$, recomendándose el uso de este a futuro. La presencia de cuerpos extraños influye en el pH del ojo, exacerbando el des- equilibrio ácido base en el ojo ${ }^{8}$, por lo que es vital extraerlos, constituyendo una emergencia oftalmológica 4 .

Este es un caso inusual de doble cuerpo extraño en fondo de saco conjuntival inferior, de acuerdo a grupo etario y evolución. Se han revisado casos de úlceras corneales y objetos intralenticulares, sin embargo, no se han reportado casos de úlcera escleral secundaria quemadura química por pila de reloj y por objetos retenidos en fondo de saco conjuntival inferior, considerando que las quemaduras químicas son más frecuentes en edad adulta y de causa laboral ${ }^{8}$. Resulta importante resaltar el difícil manejo por la evolución tórpida de la úlcera, debido al fragmento mineral alojado en el fondo de saco inferior del ojo izquierdo, y el retraso en la extracción de este considerando la presencia de un cuerpo extraño una emergencia oftalmológica.

Es importante destacar que los traumatismos oculares en la edad pediátrica son más frecuentes en los varones, están asociados a actividades de juego, ocio, accidentes domésticos ${ }^{9}$ y son evitables, por lo que la supervisión de los adultos reduce la incidencia y el nivel educativo o socioeconómico bajo aumenta el riesgo de la lesión ocular ${ }^{10}$. En este caso el motivo de consulta inicial fue el contacto ocular con detergente, y posteriormente hallazgo incidental de cuerpo extraño metálico y fragmento mineral, lo que hace cuestionar la información suministrada por los representantes del paciente, que evidencia la poca supervisión por parte de estos, y cuyas consecuencias son los accidentes y la dificultad para realizar el diagnóstico adecuado.

\section{Conclusiones}

- En casos de úlceras esclerales donde no se evidencia cicatrización adecuada, en un contexto donde el interrogatorio es escueto, el paciente es poco colaborador, y el edema con secreción no facilitan la evaluación, no se debe descartar la presencia de cuerpo extraño en fondo de saco, recomendándose el uso de la Radiografía de Órbita, para el diagnóstico inicial.

- Se recomienda el uso de antibióticos tópi- 
cos ante la presencia de cuerpos extraños metálicos y minerales, ya que son vehículos de contaminación.

- El lavado ocular es imprescindible como manejo inicial de las quemaduras por sustancias químicas, y en presencia de un cuerpo extraño constituye una emergencia oftalmológica que requiere atención para extracción de inmediato.

- Los traumatismos oculares son evitables por lo que la supervisión de los adultos reduce su incidencia.

- Este es un caso inusual de doble cuerpo extraño en fondo de saco conjuntival inferior, de acuerdo a grupo etario y evolución. Hasta los momentos no se han reportado otros casos de úlcera escleral secundaria quemadura química por pila de reloj y por objetos retenidos en fondo de saco conjuntival inferior, resaltando el arduo manejo por la evolución tórpida de la úlcera, y la demora en la extracción del cuerpo extraño considerando que es una emergencia oftalmológica.

\section{Referencias}

1.- Zambrano A, Abudi V: Traumatismos Oculares en niños. Médico Oftalmólogo; 2007: 36-38.

2.- Cilvetti A: Traumatismos Oculares. Hospital Clínico
Universitario de Málaga. Disponible en: http://www. medynet.com/usuarios/jraguilar/Manual de urgencias y Emergencias/trauocu.pdf (visitado 23 de febrero de 2011).

3.- Behrman R, Kliegman R, Jenson HN: Tratado de Pediatría. $17^{\circ}$ edición. 2006.

4.- García G, Fernández C: Traumatismos oculares en la infancia. An Pediatr Contin 2004; 2 (4): 255-8.

5.- Lima V, Caballero-Palomares M: Trauma Ocular: Distribución de acuerdo con la clasificación estandarizada. Trauma 2002; 5 (1): 5-10.

6.- Zaghen V, Monsalve E, Woodard H, Ramos N, Navas $N$ : Traumatismos oculares pediátricos que ameritaron hospitalización. Rev. Oftalmol. Venez 2005; 61 (3).

7.- Macedo Filho ET, Lago A, Duarte K, Liang SJ, Lima $A L$, Freitas D: Superficial corneal foreign body: laboratory and epidemiologic aspects. .Arq Bras Oftalmol 2005; 68 (6): 821-3.

8.- Solís J: Manejo de Quemaduras oculares por álcalis y ácidos. Revista Médica de Costa Rica y Centroamérica LXVII. 2010; 595: 515-7.

9.- Placeres J, Mobayed J, Mengual E: Traumatismos oculares en edad pediátrica. Actualización en oftalmología pediátrica Sociedad Española de Pediatría Extrahospitalaria y Atención Primaria. http://www.sepeap.org/ archivos/libros/OFTALMOLOGIA/actualizacionoftpediatrica/Ar_1_8_51_APR_1.pdf (visitado 23 de febrero de 2011)

10.- Casanova Gordó JM: Traumatismos oculares. Pediatría Integral. Volumen XIII. Número 7. Septiembre de 2009. 